\title{
LETTER
}

\section{Calculation of the normal range of extravascular lung water}

\author{
Zhongheng Zhang Jr* \\ See related research by Tagami et al., http://ccforum.com/content/14/5/R162
}

A recent article by Takashi Tagami and colleagues [1] investigated the correlation between extravascular lung water (EVLW) and postmortem lung weight, as well as the normal range of the EVLW. Since it is ethically inappropriate to conduct invasive procedures using the PiCCO monitoring system in healthy individuals, the real normal range of EVLW remains unreported. To solve this problem, the authors built a linear correlation equation from the EVLW measurements and related postmortem lung weight, then substituted the lung weight factor in the equation with normal values of lung weight reported in another large study. This is an ingenious study design. However, there is one issue worthy of consideration when interpreting the results. The population in this study was critically ill patients, including a significant number of patients with lung injury. Thus, it is not surprising that the EVLW is greater in the study population compared to the normal one, as well as the lung weight $(1,320 \mathrm{~g}$ versus $878 \mathrm{~g}$ in male or $636 \mathrm{~g}$ in female). Nevertheless, the equation was obtained from the critically ill patients with lung injury, and cannot be extrapolated to healthy individuals. That is because the proportion of EVLW in lung weight is greater in patients with lung injury than in normal lung. The correlation coefficient should be modified if the normal lung weight is substituted into the equation. Although the difference in correlation coefficients between respiratory failure and non-respiratory failure groups was not statistically significant, it can be explained by the limited sample size, which is subject to type II error.

\section{Authors' response}

Takashi Tagami and Shigeki Kushimoto

We would like to thank Dr Zhang for his thoughtful comment on our study [1]. We regret that we did not provide the number of patients with normal lung (NL) and the equation for EVLW and lung weight. We are glad to provide these details now.

Nine patients had NL and 21 had lungs heavier than NL (HL). We observed a close correlation between EVLW and postmortem lung weight in both groups (NL, $r=0.78$, $P<0.05$; HL, $r=0.83, P<0.01)$. The linear regression equations were as follows: for NL, EVLW $=0.53 \times$ Lung weight + 5.0; for HL, EVLW $=0.58 \times$ Lung weight -90.0 . The original equation for all 30 patients was EVLW = $0.56 \times$ lung weight -58.0 [1]. The correlation coefficients between the subjects was high and the equations were statistically significant for both groups. Although Dr Zhang suggested modifying the 'correlation coefficient', it is impossible and nonsensical in order to calculate EVLW

*Correspondence: zh_zhang1984@hotmail.com

Jinhua municipal central hospital, 351\#, Mingyue Street, Jinhua, Zhejiang province, P. R. China, 321000 from the equation. An equation consists of a slope and an intercept. Therefore, we provide the results comparing those parameters. There was no statistically significant difference in the slope $(P=0.50)$ and the intercept $(P=$ 0.35 ) for the equations between NL and HL, and in the slope $(P=0.60)$ and the intercept $(P=0.52)$ for the equations between NL and all 30 patients. We hope these data answer Dr Zhang's insightful comments.

\section{Abbreviations}

EVLW, extravascular lung water; HL, lungs heavier than NL; NL, normal lung.

\section{Competing interests}

The author declares that they have no competing interests.

Published: 29 October 2010

\section{References}

1. Tagami T, Kushimoto S, Yamamoto Y, Atsumi T, Tosa R, Matsuda K, Oyama R, Kawaguchi T, Masuno T, Hirama H, Yokota H: Validation of extravascular lung water measurement by single transpulmonary thermodilution: human autopsy study. Crit Care 2010, 14:R162.

doi:10.1186/cc9298

Cite this article as: Zhongheng Z Jr: Calculation of the normal range of extravascular lung water. Critical Care 2010, 14:448. 